\title{
Anaemia of Chronic Disease: An In-Depth Review
}

\author{
Anazoeze Jude Madu ${ }^{a}$ Maduka Donatus Ughasorob \\ Departments of ${ }^{a}$ Haematology and Immunology and b Paediatrics, College of Medicine, University of Nigeria, \\ Enugu, Nigeria
}

\section{Keywords}

Anaemia - Chronic disease/inflammation · Hepcidin ·

Ferritin · Pathogenesis · Cytokines tions involve the use of erythropoiesis-stimulating agents, blood transfusion, and iron supplementation, in addition to treating the underlying disease.

\begin{abstract}
Anaemia is the most common haematological disorder affecting humanity and is usually observed in chronic disease states such as non-specific anaemia, which may cause diagnostic difficulties. In chronically ill patients with anaemia, this has a negative impact on quality of life as well as survival. This paper aims at reviewing the pathogenesis of this form of anaemia with a view to suggesting future targets for therapeutic intervention. The ability to diagnose this disorder depends on the ability of the physician to correlate the possible clinical pathways of the underlying disease with the patients' ferrokinetic state. It is important to rule out iron deficiency and other causes of anaemia as misdiagnosis will in most cases lead to refractoriness to standard therapy. The cytokines and acute-phase proteins play important roles in the pathogenesis of anaemia of chronic disease. Alterations in the metabolism of iron via the molecule hepcidin and ferritin are largely responsible for the consequent anaemia. Concomitant iron deficiency might be present and could affect the diagnosis and therapeutic protocol. Treatment op-
\end{abstract}

\section{KARGER}

E-Mail karger@karger.com www.karger.com/mpp (c) 2016 S. Karger AG, Basel

Karger

This is an Open Access article licensed under the terms of the Creative Commons Attribution-NonCommercial 3.0 Unported license (CC BY-NC) (www.karger.com/OA-license), applicable to the online version of the article only. Distribution permitted for non-commercial purposes only.

\section{Introduction}

Anaemia of chronic disease (ACD) or anaemia of chronic inflammation is the most common cause of anaemia in admitted patients [1]. It is the second most prevalent cause of anaemia, after iron deficiency anaemia (IDA) [2-4]. It may be difficult to delineate the prevalence rate of this condition as it is often confused with IDA and is usually a diagnosis of exclusion. The incidence of ACD is known to increase with age, affecting $77 \%$ of the elderly in whom no clear cause of anaemia has been reported, thus indicating a multifactorial aetiology $[5,6]$. The ACD is found in patients in whom their current illness elicits an active immune/inflammatory response leading to reduced iron uptake at varying sites but not to patients in whom their illness or its treatment directly causes anaemia as is seen in some malignancies and use of cytotoxic drugs [5]. It is usually observed as a mild to moderate anaemia in patients diagnosed with other 
Table 1. Underlying causes of ACD

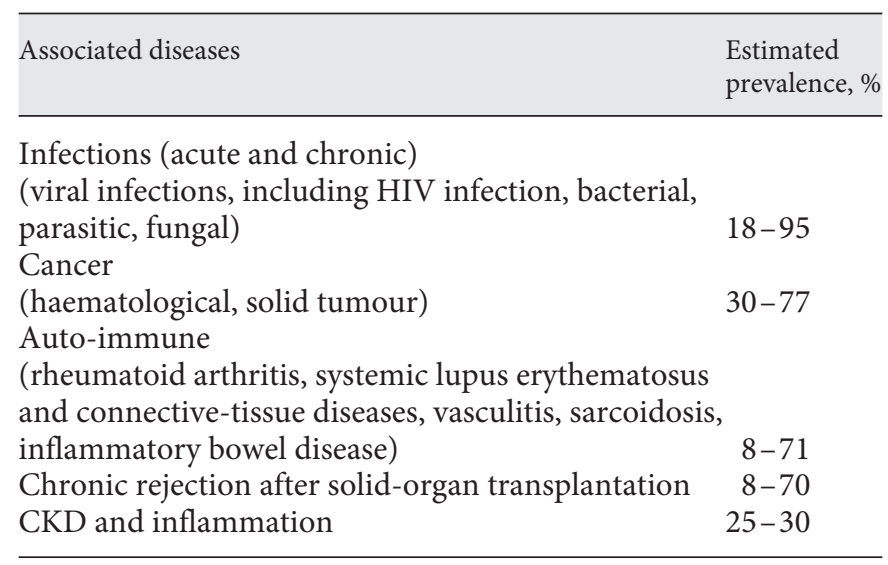

chronic disease conditions, which may not be purely inflammatory. It is seen in several disease states such as malignancies, chronic infections and auto-immune diseases $[2,4]$, thereby indicating the multiplicity in pathogenetic pathways that may lead to ACD. A list of the possible causes of ACD is shown in Table 1.

The pathogenetic processes involved reflect the active protection expressed by an effective immune system in depriving the invading cells of iron, which is an important nutrient for the proliferation of both cancer cells and pathogens. These processes primarily include bone marrow invasion by tumours or infective agents, alteration of iron metabolism and diversion of body iron, haemophagocytosis, reduction in erythropoiesis, and diminished response to erythropoietin stimulation $[1,5,6]$. Though not completely understood, the pathogenetic processes are thought to be mediated through the actions of tumour necrosis factor (TNF) and interleukins (IL)-1 and -6 , and interferon (IFN) [7]. These cytokines, as well as the acute-phase protein hepcidin, are believed to inhibit iron release from the marrow macrophages to the budding erythroid progenitors. The cytokines also directly induce the modulation of translation/transcriptions of genes involved in iron homeostasis, either directly or via production of labile radicals [5]. The diagnosis of ACD which requires a good knowledge of the processes that affect production of the key molecules and interpretation of the results of the circulating iron status parameters as well as ferritin and hepcidin assays is an important step in distinguishing ACD from IDA [8].

A diagnosis of ACD does not suffice for anaemia observed in all chronically ill patients [9], because of the temptation to label all ill patients in whom a definite cause of anaemia cannot be discerned as ACD. A diligent effort should be made to unravel the underlying cause of anaemia in all patients as well as to rule out iron deficiency. The success of management of either condition is therefore reliant on making a correct diagnosis, as each would require different treatment protocols $[8,10]$. In addition to treating the underlying condition, targeting other inflammatory pathways may be beneficial to achieve a rapid resolution of anaemia $[11,12]$. This review delved into the distinct pathogenetic causes of ACD, with some emphasis on future management possibilities.

\section{Pathogenesis of ACD}

The pathogenetic mechanisms vary [1] as each of the pathways depends largely on the aetiological cause. However, each of the individual factors plays some role in the eventual cause of anaemia.

\section{Iron Dysregulation/Reticulo-Endothelial Iron Block}

This is a major observation in ACD, thus the eventual occurrence of a hypochromic microcytic red cell picture similar to what is found in iron deficiency. Microbial invasion, malignancies and auto-immune disorders cause activation of CD3 T lymphocytes and macrophages which release cytokines - IFN- $\gamma$ (from T cells), TNF- $\alpha$, IL- 1 and -6 (from monocytes) $[13,14]$. TNF- $\alpha$ is secreted also by neutrophils, macrophages, T cells, and natural killer cells in response to stimulation by IL-2, granulocyte-monocyte colony-stimulating factor, platelet-associated factor and is inhibited by IL-6, transforming growth factor, and prostaglandin $\mathrm{E}_{2}$ [15]. Bacterial lipopolysaccharides and IL-6 induce the hepatic cells to release hepcidin [16], which enhances the breakdown of ferroportin, leading to blockade of the duodenal iron transfer $[17,18]$. Both molecules also upregulate the expression of divalent metal transporter (DMT-1), which is a transmembrane protein involved in the uptake of iron by macrophages [12]. They also inhibit the expression (initiate internalization) of ferroportin 1, which depresses the export of iron from the macrophages to the growing erythroid precursors; this action is influenced by hepcidin. The common aetiological causes of ACD are shown in Table 1.

Erythropoiesis is inhibited by several cytokines including IFN- $\gamma$, transforming growth factor- $\beta$ ), and TNF- $\alpha$. The action of these cytokines is mediated via the p38 mitogen-activated protein kinase pathway, while IFN- $\gamma$ acts via the Janus-associated kinase (JAK/STAT) pathway [19]. Activation of this pathway leads to production of 
Table 2. Effects of cytokines on iron metabolism and erythropoiesis

\begin{tabular}{ll}
\hline Cytokines & Effects on red cell dynamics \\
\hline TNF- $\alpha$ inhibition of erythropoietin production & $\begin{array}{l}\text { Stimulation of ferritin synthesis } \\
\text { Enhances degradation and phagocytosis of effete red cells } \\
\text { Direct inhibition of erythropoiesis }\end{array}$ \\
\hline IFN- $\gamma$ inhibits production of erythropoietin & $\begin{array}{l}\text { Increases intracellular iron by stimulation of DMT-1 and inhibition } \\
\text { of ferroportin } \\
\text { Increased nitric oxide production and inducible nitric oxide synthase } \\
\text { mRNA expression }\end{array}$ \\
\hline IL-6 increases iron uptake via DMT-1 activation & $\begin{array}{l}\text { Reduces TfR by decreasing TfR RNA expression } \\
\text { Downregulates expression of SLC4a1 in erythroid precursors }\end{array}$ \\
\hline
\end{tabular}

IL-4 and -10 increase ferritin via action on iron regulatory elements/proteins

intracellular factors which enhance apoptosis by regulating transcription and eventual myelosuppression. IFN- $\gamma$ stimulates ferritin transcription on the one hand and inhibits transferrin receptor (TfR) mRNA expression via an iron-regulatory element-protein-independent process [20-22]. It also increases expression of DMT-1, which is involved in the active transport of ferrous molecules from the lumen to the duodenal endothelial cell cytoplasm [21]. Hence, the absorbed iron is retained in the intestinal endothelial cells which are eventually shed while serum ferritin levels remain elevated despite an apparent scarcity. The marrow macrophages, though having an increased uptake of iron, retain their cytoplasmic iron while the budding erythroid precursors remain deprived.

Hepcidin is a "hairpin"-like molecule produced by the liver and inhibits iron absorption from the duodenum as well as release of iron by the bone marrow macrophages $[17,23]$. This action is mediated via regulating intestinal iron absorption [24] as well as plasma and tissue iron concentration by its ability to degrade its receptor ferroportin [25]; this action is not restricted to the macrophages, but also occurs in the duodenal enterocytes. Hepcidin downregulates intestinal transepithelial iron transport by causing an ubiquitin-dependent proteosome degradation of intestinal DMT-1 [26]. The effect on DMT-1 and ferroportin occurs by internalizing and degrading these membrane receptors and, thus, inhibits the release of iron by the macrophages while reducing absorption of iron by the intestinal mucosal cells $[1,27]$. This is actually a defence mechanism that the body adopts to produce a hypoferraemic state, thus denying bacterial and cancer cells their much-needed iron. This, as well as the pathway involving IL-6, is thought to be the major mechanism in the occur- rence of ACD. A list of the major cytokines involved in ACD and their mechanisms of effecting these actions is given in Table 2.

\section{Reduced Erythropoiesis}

IL- 6 has been proposed to be the most important cytokine mediating the pathogenesis of ACD [16]. It is a potent inhibitor of TNF- $\alpha$ and induces the transcription of ferritin that leads to increased retention and iron storage within the reticulo-endothelial cells. Previous studies had suggested the existence of other pathways of anaemia induction in chronically ill animal species, in which suppression of TNF elevation did not prevent the occurrence of anaemia [11, 28, 29]. IL-6 inhibits erythropoiesis through other pathways other than the inhibition of absorption and uptake of iron [18]. It downregulates the expression of the SLC4al gene in late erythroid precursors and thereby reduces haemoglobin synthesis [14]. It also reduces the mitochondrial mass and function, in the budding erythroid progenitors. It induces an increase in the hepatic synthesis of the acute-phase protein hepcidin. IL-6 seems to cause anaemia through other pathways that are not hepcidin dependent [30], thus suggesting either direct inhibition of erythropoiesis or the existence of other yet to be established pathways.

Activin B production by hepatic cells is markedly increased during inflammation and binds to the bone morphogenetic protein (BMP) receptor type 1 . Activation of this receptor acts via the SMAD and JAK-STAT transmembrane proteins to cause upregulation of hepcidin expression [31]. Both IL-6 and hepatic hepcidin expression have been found to be significantly increased in various malignancies [23]. 
Hepcidin inhibits the export of iron from the enterocytes, hepatocytes, and marrow macrophages [10, 32], thereby creating a hypoferraemic environment, and thus denies the invading microbes as well as the erythroid cells their much-needed iron. IFN- $\gamma$ induces apoptosis in erythroid precursors by increasing nitric oxide production and inducible nitric oxide synthase mRNA production [22]. IFN- $\gamma$ and to a lesser extent IFN- $\alpha$ and $-\beta$ have been observed to induce apoptosis of the erythroid burst-forming and colony-forming units. This action is mediated in part via the action of ceramide and due to reduction in erythropoietin receptors in precursor erythroid cells [5]. Other modalities of IFN action include reduction in quantity and activity of erythropoietin as well as reduced expression of other growth factors such as stem cell factor.

\section{Diminished Response to Erythropoietin}

In some cases of $\mathrm{ACD}$, the erythropoietin response (level) is not commensurate to the degree of anaemia, thus the term "blunted erythropoietin response." This phenomenon had been observed in sickle cell patients with chronic kidney disease (CKD), where erythropoiesis-stimulating agents (ESAs) do not correct the anaemia. This can be partly explained by the fact that the cytokines, bacterial lipopolysaccharides and IFN- $\gamma$ [33] induce formation of nitric oxide and oxygen free radicals, which directly inhibit expression of erythropoietin in vitro [34]. These reactive oxygen species are also thought to inhibit the erythropoietin-inducing transcription factors as well as to damage the erythropoietin-producing cells. Silymarin, which modulates immune cells by inhibiting prostaglandin and prostacyclin production as well as neutrophil and monocyte activation and mobilization, has the capacity to reverse this trend [34].

A blunted response to erythropoietin also occurs in a quarter of patients with end-stage renal disease, necessitating the use of higher doses [18]. Several inflammatory proteins as well as the inflammatory cytokine, especially IL-6, had been linked with this diminishing response to erythropoietin. In some patients with CKD and suboptimal response to erythropoietin, vitamin D therapy leads to improved anaemia with reduced requirement for erythropoietin. This response is reportedly present in patients in whom there was no depletion in parathyroid hormone levels. Calcitriol had been observed to directly increase proliferation of erythroid precursors via the activation of $1 \alpha$-hydroxylase $[35,36]$. Improvement in anaemia as well as response to ESAs had also been observed with vitamin A, D, and E supplementation [3537]. Activation of vitamin $D$ receptors on immune cells leads to release of IL-10 from the bone marrow stromal and accessory cells. Recent studies have shown that 1,25-dihydroxyvitamin $\mathrm{D}_{3}$ downregulates hepcidin and increases ferroportin expression in lipopolysaccharidestimulated THP-1 cells. High doses of vitamin D have been found to decrease the prohepcidin cytokines IL-6 and IL- $1 \beta$ in a dose-dependent manner [34]. This has the effect of inducing proliferation of erythroid progenitor cells as well as inhibiting inflammation [35]. Also erythroferrone, a regulator of hepcidin synthesis and iron homeostasis, has been found to downregulate the expression of hepcidin mRNA in thalassaemic mice. Ablation of the erythroferrone gene, Erfe, ameliorates the iron overload and restores hepcidin levels in this situation [35]. This indicates that anaemia in CKD with background inflammation has multiple pathogenetic pathways and may require further investigation if treatment with dialysis and ESA does not yield optimal recovery from anaemia.

\section{Hypoferraemia and Reduced Erythrocyte Survival}

IFN- $\gamma$ and bacterial lipopolysaccharides upregulate the expression of DMT-1 in a dose-dependent manner [38], thereby enhance the uptake of unbound iron into the enterocytes and the monocyte/macrophages, and cause retention of iron within the monocytes by downregulating the expression of ferroportin mRNA (a putative iron exporter). In chronicaly ill patients, higher levels of TNF- $\alpha$ and IL- 6 had both been reported to correlate with lower levels of serum iron [39], thus creating a prevailing atmosphere of hypoferraemia with reduction in the erythropoietic capacity. This may also be due to an increased rate of erythrophagocytosis, a process designed to remove senescent and damaged red cells in normal situations, this damage being caused by cytokines, endotoxins, and reactive oxygen species. Some animal experiments have revealed that sublethal doses of TNF- $\alpha$ may cause phagocytosis of erythrocytes by macrophages.

Hypoxia leads to increased transcription of hepcidin mRNA [40]. This process is thought to be mediated through platelet-derived growth factor [41]. Inversely, the production of free oxygen radicals in inflammation causes release of pro-inflammatory cytokines including hepcidin. Other pro-inflammatory cytokines like IFN- $\gamma$ cause increased expression of inducible nitric oxide mRNA and the subsequent production of nitric oxide [22]. This in conjunction with the production of superoxide molecules in inflammation may cause reversal of the effects of hypoxia on hepcidin production. This induces nitric oxide-mediated apoptosis of red cell precursors and thus worsens anaemia. 


\section{Bone Marrow Infiltration}

Anaemia secondary to cancers occurs via 3 basic mechanisms: (a) reduced production of red cells (either by tumour invasions, effect of cytotoxic drugs, suboptimal nutrition, or cytokine-based inhibition), (b) increased red cell loss (haemolysis or haemorrhage), and (c) miscellaneous aetiologies [9]. In most cases there are significant overlaps in these mechanisms; however, the major mechanism is cancer-driven inflammation [42]. Marrow invasion by malignant cells leading to physical obstruction and destruction of the bone marrow microenvironment seems to occur with time in most cancers. However, in some cases of malignancies, significant anaemia is observed in the absence of marrow invasion or scarcity of vital nutrients. This signifies that other pathways may be important in the pathogenetic processes leading to anaemia in cancers. Tumors cause secretion of cyclo-oxygenase-2, as well as vascular endothelial growth factor, granulocyte-monocyte colony-stimulating factor, IL-6, and TNF- $\alpha$, which lead to cancer cachexia and anaemia [43]. Celecoxib, a cyclo-oxygenase-2 inhibitor, has also been noted to counteract the anaemia and cachexia associated with ACD [43]. The point of action of several cytokines involved in ACD is shown in Table 2.

Growth differentiation factor 15 (GDF-15) is an inhibitor of leucocyte integrin, and a member of the transforming growth factor- $\beta$ superfamily [44]. This novel molecule has been observed to have an inverse relationship with serum hepcidin levels in cancer patients with ESA-resistant anaemia. Elevation of GDF-15 results in depression of hepcidin levels [45] and is associated with tumour metastasis, angiogenesis, progression and haemopoiesis $[45,46]$. This has been proposed to be a major mechanism of anaemia in cancer-driven inflammation, as the serum level of GDF-15 is correlated to a large extent with the degree of anaemia in cancer patients [45].

In the case of infective agents like Plasmodium and HIV, toxic products of these parasites directly suppress erythropoiesis. These organisms and malignant cells also competitively deprive the erythroid precursors of available iron. The invading microbial cells require iron as an important component of several iron-containing enzymes needed for protein synthesis and proliferation.

\section{Laboratory Diagnosis}

Initial assessment involves a good history of the illness [9] as well as general investigations to rule out other causes of anaemia. Review of the morphology of the blood film and bone marrow, reticulocyte count, stool analysis, serum bilirubin and lactate dehydrogenase assay, and assessment of renal function are required. Important aspects in the diagnosis of ACD are differentiating it from IDA and other causes of hypochromic, microcytic anaemia. This requires an in-depth understanding of the stimuli and regulatory pathways of production of the various molecules that are usually assayed.

\section{Blood Film Morphology}

The initial red cell morphology shows a normochromic and normocytic picture; this changes with time to become hypochromic and microcytic. The haemoglobin concentration is usually $8-9.5 \mathrm{~g} / \mathrm{dL}$ (mild to moderate anaemia), and rarely drops to below $6 \mathrm{~g} / \mathrm{dL}$. In cases of suspected ACD with severe anaemia, other causes of red cell loss or destruction should be vigorously investigated. The reticulocyte count (or better still the reticulocyte index) is usually reduced in ACD as well as in IDA. The blood film may provide information on the underlying cause of ACD: thrombocytosis in cases of chronic haemorrhage, toxic granules in neutrophils in severe sepsis, hypersegmented neutrophils in mixed nutritional deficiency or folate $/ \mathrm{B}_{12}$ deficiency found in malignant conditions.

\section{Ferrokinetic Studies}

The major difference between ACD and IDA is that in IDA there is an absolute lack (serum ferritin below $30 \mathrm{ng} /$ $\mathrm{mL}$ ) [1] of iron, while the pathogenesis of ACD is multifactorial and the iron though available is not accessed by the young erythroid precursors.

In ACD, transferrin is increased while serum iron and transferrin saturation are reduced, while the erythrocytefree protoporphyrin, serum ferritin, and marrow-stainable iron are increased [47]. In ACD, the reduced transferrin saturation is not only due to lack of iron, but also due to increased production of transferrin. The hypoferraemia is due to trapping of iron in the cells of the reticulo-endothelial system, and the low serum iron consequently results in low transferrin saturation. The level of the transport protein transferrin is therefore increased in IDA but reduced or normal in ACD [1].

\section{Serum Ferritin/Ferritin Receptor}

Ferritin is an acute-phase protein, and its levels are increased in chronic inflammatory states. It is a good measure of body iron status, once underlying inflammation has been ruled out. The normal levels of serum ferritin are usually between 15 and $300 \mu \mathrm{g} / \mathrm{L}$. Therefore in patients with ACD serum ferritin is usually increased [48] due to 
Table 3. Serum levels that differentiate ACD from IDA

\begin{tabular}{llll}
\hline Variable & ACD & IDA & Both conditions \\
\hline Iron & Reduced & Reduced & Reduced \\
Serum transferrin & Reduced to normal & Increased & Reduced \\
Transferrin saturation & Reduced & Reduced & Reduced \\
Ferritin & Normal to increased & Reduced & Reduced to normal \\
Soluble TfR & Normal & Increased & Normal to increased \\
Ratio of TfR to log ferritin & Low $(<1)$ & High $(>2)$ & High $(>2)$ \\
Cytokine levels & Increased & Normal & Increased \\
\hline
\end{tabular}

retention of iron by the reticulo-endothelial cells and increased production secondary to inflammation.

The soluble TfR (sTfR) is a broken-off fragment of the membrane TfR, whose levels seem to correlate with the TfR [32]. The production of both molecules is regulated by the availability of intracellular iron via the iron regulatory element-protein interaction. Therefore low iron availability leads to increased TfR and sTfR. Though the level of sTfR is also affected by inflammatory cytokines, it is usually useful in differentiating ACD from IDA [4]. This is because the pro-inflammatory cytokines and the erythroid iron deficiency both affect the sTfR in opposite directions, therefore balancing out their impact. The result is that the sTfR in ACD is usually lower than in IDA and similar to the values in non-anaemic patients.

\section{Hepcidin}

This is a "hairpin"-like 25-amino acid chain protein, produced in the hepatic parenchymal cells. This structure is maintained by the disulphide bonds which exist between the 4 cysteine amino acid residues in the chain. It is also produced to some extent by activated neutrophils and macrophages. Hepcidin synthesis is induced by iron overload and inflammation, and inhibited by anaemia and hypoxia [40]. It acts by inhibiting export of iron from the iron-containing cells - duodenal endothelial cells and macrophages. This action is mediated via the effect on ferroportin, which induces its breakdown/degradation. Ferroportin is the sole iron exporter in iron-containing cells. This is thought to be the main pathogenetic mechanism of ACD. Serum hepcidin is currently being proposed as the most accurate serological marker for the differentiation of ACD and IDA [49]. The important laboratory differences between ACD and IDA as well as the expected results in situations where both coexist are listed in Table 3.

\section{Erythropoietin Assay}

Erythropoietin is produced by the renal medullary cells in response to anaemia as well as reduced oxygen tension. The assay results in ACD must be interpreted with caution, and its main use may be its predictive value of a response to erythropoietin therapy. After recombinant human erythropoietin treatment for 2 weeks, an erythropoietin level of $>100 \mathrm{U} / \mathrm{L}$ or a ferritin level of $>400$ $\mathrm{ng} / \mathrm{mL}$ is predictive of a poor response to erythropoietin in $88 \%$ of patients not receiving concomitant anticancer drugs [47]. This has not been replicated in patients receiving cancer chemotherapy.

\section{Treatment}

The goals of treatment in ACD include: improve oxygen-carrying capacity of blood, and detect and treat the underlying cause. In a situation where end organ damage may be imminent and overactive cardiac compensatory mechanisms may lead to deleterious consequences, urgent treatment obviates the poor prognostic outcome associated with anaemia in most disease conditions. Anaemia has been observed to confer a poorer prognosis in patients with CKD, cancers, and congestive cardiac failure. Haemoglobin levels of $\leq 8 \mathrm{~g} / \mathrm{dL}$ in CKD patients on haemodialysis are associated with a double increase in the risk of death compared with patients who had a haemoglobin level of 10-11 g/dL [48]. Patients who had their haemoglobin concentration optimized to between 10 and $11 \mathrm{~g} / \mathrm{dL}$ also had improved survival and a better treatment outcome $[49,50]$.

\section{Iron Therapy}

Iron therapy may not be very effective in $\mathrm{ACD}$ bearing in mind that the pathogenesis involved is a relative deficiency in availability of iron to the red cell precursors and 
not an absolute deficiency. In addition, several micro-organisms and tumour cells utilize the excess iron for their cellular proliferation. Iron is known to have an inhibitory effect on the immune system by downregulating IFN- $\gamma$ mediated pathways $[50,51]$. It also enhances the production of hydroxyl radicals which cause tissue and endothelial damage.

However, in situations of poor oral intake or deficient absorption, parenteral iron may be used. Otherwise oral iron is well absorbed, has a good bio-availability in its reduced form and should be used except in proven cases of malabsorption $[52,53]$. Iron can also be administered parenterally, and this may be via intravenous and intramuscular routes. Intravenous iron is usually administered as a loading dose and given as iron dextran in a slow infusion. Its major side effect is the severe and sometimes life-threatening anaphylactic reaction that may occur, thus the need to have an emergency tray nearby [54]. Jectofer, a brand of intramuscular iron, is sometimes used but this needs to be given as a deep intramuscular injection using a Z (zig zag) technique. This is to avert the undesired aftermath of leaving a dark patch around the injection site, especially in fair-skinned individuals.

Patients with inflammatory bowel disease and CKD have all been known to benefit from oral or parenteral iron, given with ESAs [55]. This may be founded on the background that some degree of absolute iron deficiency may exist in patients with ACD; also by the inhibitory effect of iron on TNF- $\alpha$, it confers a better outcome in rheumatoid arthritis and CKD $[12,16]$.

Iron therapy should therefore be considered in patients with ACD who also have an absolute deficiency of iron and may be tried on individuals who are not responding to erythropoietin, as functional iron deficiency may be involved.

\section{Red Cell Transfusion}

Blood transfusion remains an important short-term management option especially in individuals with severe anaemia (haemoglobin $<6.5 \mathrm{~g} / \mathrm{dL}$ ) associated with cardiac decompensation. However, long-term use of red cell transfusion has been associated with increased mortality, mainly due to iron overload and immune activation by HLA antigens in patients who may be eventually transplanted [51].

\section{Erythropoiesis-Stimulating Agents}

Erythropoietin stimulates proliferation of erythroid precursor burst-forming unit erythroid in the marrow via its action on the bone morphogenetic protein (BMP-
SMAD) $[10,56]$ and JAK-STAT5 pathway. BMP is a member of the transforming growth factor- $\beta$ family which binds to serine-threonine kinase receptors and transduces signals via the SMAD. In hepatocytes BMP binds to haemojuvelin which acts as its signalling component and thus induces expression of hepcidin, with a consequent decrease in iron absorption [56]. Induction of the JAK-STAT pathway indirectly inhibits the pro-inflammatory effects of hepcidin by inhibiting IL- 6 production by monocytes [57]. Erythropoietic agents have been approved for use in ACD due to cancers or anticancer chemotherapy as well as in patients with CKD and HIV infection [47]. The mode of action involves counteracting the antiproliferative effects of the pro-inflammatory cytokines, as well as stimulation of iron uptake and haem biosynthesis in erythroid precursors. These agents should be used in conjunction with iron, and the target haemoglobin should be between 11 and $12 \mathrm{~g} / \mathrm{dL}$; previous studies had shown that levels above this were associated with a poorer treatment outcome. However, the common side effects of erythropoietin should be assessed in each patient and include raised blood pressure, cerebral convulsion/hypertensive encephalopathy, thrombo-embolic complications, iron deficiency, and influenza-like syndrome [54].

A background knowledge of ferrokinetics and the dynamics of the molecules involved in iron metabolism is necessary for the investigation, interpretation of results, and optimal management of patients with ACD. Further studies should be directed at the inhibition of hepcidin and other pro-inflammatory cytokines, in a bid to overcome their inhibitory effects on the reticulo-endothelial cells, and also at the use of molecules which are able to bind iron regulatory elements and proteins and therefore tilt the balance towards what is usually observed in IDA.

\section{Targetting the Cytokines}

$\omega-3$ poly-unsaturated fatty acids have been observed in several clinical scenarios to downregulate the production of TNF- $\alpha$ and IL- 6 . They have also shown promise in the treatment of ACD in rheumatoid arthritis and diabetes mellitus, an effect largely mediated via their action on the transcription factor nuclear factor- $\mathrm{kB}$. The polyunsaturated fatty acids have the advantage of being safe with little or no known adverse effects. The mitogen-activated protein kinase pathway which has been observed to mediate myelosuppression should attract more attention as previous molecules inhibiting the pathway have shown some promise in patients with myelodysplastic syndrome [19]. 
Hepcidin provides an interesting target in the management of ACD due to its central pathogenetic role $[8,27]$. High levels of hepcidin have been found to correlate with failure of ESAs, thus indicating that inhibition of hepcidin might improve the response to erythropoietin. A novel antihepcidin agent (NOX-H94) has been produced which leads to resolution of anaemia in animal studies [58]. Combined treatment with hepcidin inhibitors and ESAs was found to be superior to treatment with ESAs alone in animal models [8]. GDF-15 has also been proposed as a suitable target due to its central role in mutagenesis, angiogenesis, and metastasis, as well as ACD in various malignancies [46]. The idea of combination therapy has become more appealing since the revelation of a hepcidin-independent pathogenetic pathway for the development of ACD [30].

Other therapeutic targets will include the inhibition of the expression of the genes coding for the pro-inflammatory hormone hepcidin. However, more studies will be required to elucidate its protective roles in order to be able to predict the consequences of its inhibition. The genes coding for the fatty acid desaturases which are the rate-limiting enzymes in the synthesis of these $\omega-3$ fatty acids also present another interesting target. This will have a more specific impact, especially in patients suffering from terminal chronic diseases.

\section{Conclusion}

The understanding and consequent diagnosis of $\mathrm{ACD}$ has continually fazed many physicians. The actions of several pro- and anti-inflammatory cytokines and hormones produce the suppression of erythropoiesis. The majority control iron release from the intestinal mucosa and marrow macrophages via ferroportin. Future treatment options should be targeted at the genes coding these cytokines as well as using anti-inflammatory food supplements: fatty acids and vitamin D. The use of iron supplementation in conjunction with ESAs may also be beneficial.

\section{Disclosure Statement}

There is no conflict of interest.

\section{References}

1 Poggiali E, Migone De Amicis M, Motta I: Anemia of chronic disease: a unique defect of iron recycling for many different chronic diseases. Eur J Intern Med 2014;25:12-17.

2 Weiss G: Pathogenesis and treatment of anaemia of chronic disease. Blood Rev 2002;16: 87-96.

3 Weiss G, Goodnough LT: Anemia of chronic disease. N Engl J Med 2005;352:1011-1023.

4 Means RT Jr: Recent developments in the anemia of chronic disease. Curr Hematol Rep 2003;2:116-121.

5 Weiss G: Iron metabolism in the anemia of chronic disease. Biochim Biophys Acta 2009; 1790:682-693.

6 Weiss G, Goodnough LT: Anemia of chronic disease. N Engl J Med 2005;352:1011-1023.

7 Zupanic K, Sucic M, Bekic D: Anemia of chronic disease: illness or adaptive mechanism. Acta Clin Croat 2014;53:348-354.

8 Theurl M, Nairz M, Schroll A, et al: Hepcidin as a predictive factor and therapeutic target in erythropoiesis-stimulating agent treatment for anemia of chronic disease in rats. Haematologica 2014;99:1516-1524.

9 Gaspar BL, Sharma P, Das R: Anemia in malignancies: pathogenetic and diagnostic considerations. Hematology 2015;20:18-25.
10 Gangat N, Wolanskyj AP: Anemia of chronic disease. Semin Hematol 2013;50:232-238.

11 Capocasale RJ, Makropoulos DA, Achuthanandam R, et al: Myelodysplasia and anemia of chronic disease in human tumor necrosis factor-alpha transgenic mice. Cytometry A 2008; 73:148-159.

12 De Lima GA, Mazzali M, Gentil AF, et al: Anemia in chronic renal disease: evaluation of inflammatory activity on erythropoiesis and iron metabolism in patients not submitted to dialysis treatment. Clin Lab 2012;58:695-704.

13 Moldawer LL, Marano MA, Wei H, et al: Cachectin/tumor necrosis factor-alpha alters red blood cell kinetics and induces anemia in vivo. FASEB J 1989;3:1637-1643.

14 McCranor BJ, Kim MJ, Cruz NM, et al: Interleukin-6 directly impairs the erythroid development of human TF-1 erythroleukemic cells. Blood Cells Mol Dis 2014;52:126-133.

15 Gifford GE, Duckworth DH: Introduction to TNF and related lymphokines. Biotherapy 1991;3:103-111.

16 Galushko EA: The clinical significance of hepcidin detection in the patients with anemia and rheumatoid arthritis (in Russian). Klin Med (Mosk) 2014;92:21-27.

17 Nemeth E, Valore EV, Territo M, et al: Hepcidin, a putative mediator of anemia of inflammation, is a type II acute-phase protein. Blood 2003;101:2461-2463.
18 Raj DS: Role of interleukin-6 in the anemia of chronic disease. Semin Arthritis Rheum 2009; 38:382-388.

19 Katsoulidis E, Li Y, Yoon P, et al: Role of the p38 mitogen-activated protein kinase pathway in cytokine-mediated hematopoietic suppression in myelodysplastic syndromes. Cancer Res 2005;65:9029-9037.

20 Lortz S, Schroter S, Stuckemann V, et al: Influence of cytokines on DMT1 iron transporter and ferritin expression in insulin-secreting cells. J Mol Endocrinol 2014;52:301-310.

21 Costa E, Fernandes J, Ribeiro S, et al: Aging is associated with impaired renal function, INFgamma induced inflammation and with alterations in iron regulatory proteins gene expression. Aging Dis 2014;5:356-365.

22 Kheansaard W, Mas-Oo-di S, Nilganuwong S, et al: Interferon-gamma induced nitric oxidemediated apoptosis of anemia of chronic disease in rheumatoid arthritis. Rheumatol Int 2013;33:151-156.

23 Vokurka M, Krijt J, Vavrova J, et al: Hepcidin expression in the liver of mice with implanted tumour reacts to iron deficiency, inflammation and erythropoietin administration. Folia Biol (Praha) 2011;57:248-254. 
24 Becker C, Orozco M, Solomons NW, et al: Iron metabolism in obesity: how interaction between homoeostatic mechanisms can interfere with their original purpose. I. Underlying homoeostatic mechanisms of energy storage and iron metabolisms and their interaction. J Trace Elem Med Biol 2015;30:195-201.

25 Ganz T, Nemeth E: Hepcidin and iron homeostasis. Biochim Biophys Acta 2012;1823: 1434-1443.

26 Brasse-Lagnel C, Karim Z, Letteron P, et al: Intestinal DMT1 cotransporter is down-regulated by hepcidin via proteasome internalization and degradation. Gastroenterology 2011;140:1261-1271. e1261.

27 Sun CC, Vaja V, Babitt JL, et al: Targeting the hepcidin-ferroportin axis to develop new treatment strategies for anemia of chronic disease and anemia of inflammation. Am J Hematol 2012;87:392-400.

28 Schubert TE, Echtenacher B, Hofstadter F, et al: Failure of interferon-gamma and tumor necrosis factor in mediating anemia of chronic disease in a mouse model of protracted septic peritonitis. Int J Mol Med 2005;16:753758.

29 Kumar M, Bhoi S: Cytokines, granulocytemonocyte colony stimulating factor, interleukin-3 and erythropoietin: can they be a therapeutic option for the stimulation of hematopoietic progenitor cells in trauma-hemorrhagic shock? Indian J Crit Care Med 2016; 20:207-209.

30 Langdon JM, Yates SC, Femnou LK, et al: Hepcidin-dependent and hepcidin-independent regulation of erythropoiesis in a mouse model of anemia of chronic inflammation. Am J Hematol 2014;89:470-479.

31 Besson-Fournier C, Latour C, Kautz L, et al: Induction of activin $\mathrm{B}$ by inflammatory stimuli up-regulates expression of the iron-regulatory peptide hepcidin through $\operatorname{Smad} 1 / 5 / 8$ signaling. Blood 2012;120:431-439.

32 Masson C: Rheumatoid anemia. Joint Bone Spine 2011;78:131-137.

33 De Lurdes Agostinho Cabrita A, Pinho A, Malho A, et al: Risk factors for high erythropoiesis stimulating agent resistance index in pre-dialysis chronic kidney disease patients, stages 4 and 5. Int Urol Nephrol 2011;43:835840 .

34 Nazemian F, Karimi G, Moatamedi M, et al: Effect of silymarin administration on TNFalpha serum concentration in peritoneal dialysis patients. Phytother Res 2010;24:16541657.
35 Icardi A, Paoletti E, De Nicola L, et al: Renal anaemia and EPO hyporesponsiveness associated with vitamin $\mathrm{D}$ deficiency: the potential role of inflammation. Nephrol Dial Transplant 2013;28:1672-1679.

36 Bel'mer SV, Mitina EV, Karpina LM, et al: Iron deficiency anemia and anemia in chronic celiac disease in children. Eksp Klin Gastroenterol 2014;23-29.

37 Panichi V, Rosati A, Paoletti S, et al: A vitamin E-coated polysulfone membrane reduces serum levels of inflammatory markers and resistance to erythropoietin-stimulating agents in hemodialysis patients: results of a randomized cross-over multicenter trial. Blood Purif 2011;32:7-14.

38 Ludwiczek S, Aigner E, Theurl I, et al: Cytokine-mediated regulation of iron transport in human monocytic cells. Blood 2003;101: 4148-4154.

39 Lopez-Prieto J, Gonzalez-Reimers E, Aleman-Valls MR, et al: Iron and proinflammatory cytokines in chronic hepatitis $C$ virus infection. Biol Trace Elem Res 2013;155:5-10.

40 Ravasi G, Pelucchi S, Greni F, et al: Circulating factors are involved in hypoxia-induced hepcidin suppression. Blood Cells Mol Dis 2014;53:204-210.

41 Sonnweber T, Nachbaur D, Schroll A, et al: Hypoxia induced downregulation of hepcidin is mediated by platelet derived growth factor BB. Gut 2014;63:1951-1959.

42 Zhang S, Chen Y, Guo W, et al: Disordered hepcidin-ferroportin signaling promotes breast cancer growth. Cell Signal 2014;26: 2539-2550.

$43 \mathrm{Xu}$ X, Jiang M, Zhang Y, et al: Celecoxib attenuates cachectic events in mice by modulating the expression of vascular endothelial growth factor. Mol Med Rep 2015;11:289294.

44 Mei S, Wang H, Fu R, et al: Hepcidin and GDF15 in anemia of multiple myeloma. Int J Hematol 2014;100:266-273.

45 Jiang F, Yu WJ, Wang XH, et al: Regulation of hepcidin through GDF-15 in cancer-related anemia. Clin Chim Acta 2014;428:14-19.

46 Unal B, Alan S, Bassorgun CI, et al: The divergent roles of growth differentiation factor- 15 (GDF-15) in benign and malignant skin pathologies. Arch Dermatol Res 2015;307:551557.
47 Spivak JL: Iron and the anemia of chronic disease. Oncology (Williston Park) 2002;16:2533.

48 Przybyszewska J, Zekanowska E, KedzioraKornatowska K, et al: Serum prohepcidin and other iron metabolism parameters in elderly patients with anemia of chronic disease and with iron deficiency anemia. Pol Arch Med Wewn 2013;123:105-111.

49 Shu T, Jing C, Lv Z, et al: Hepcidin in tumorrelated iron deficiency anemia and tumor-related anemia of chronic disease: pathogenic mechanisms and diagnosis. Eur J Haematol 2015;94:67-73

50 Nairz M, Haschka D, Demetz E, et al: Iron at the interface of immunity and infection. Front Pharmacol 2014;5:152.

51 Kennedy RB, Ovsyannikova IG, Haralambieva $\mathrm{IH}$, et al: Genome-wide SNP associations with rubella-specific cytokine responses in measles-mumps-rubella vaccine recipients. Immunogenetics 2014;66:493-499.

52 Blumenstein I, Dignass A, Vollmer S, et al: Current practice in the diagnosis and management of IBD-associated anaemia and iron deficiency in Germany: the German AnaemIBD Study. J Crohns Colitis 2014;8:1308-1314.

53 Derovs A, Pokrotnieks J, Derova J, et al: Current opinion on the management of iron deficiency anaemia in gastrointestinal diseases. Eksp Klin Gastroenterol 2014;97-105.

54 Herfs R, Fleitmann L, Kocsis I: Treatment of iron deficiency with or without anaemia with intravenous ferric carboxymaltose in gynaecological practices - a non-interventional study. Geburtshilfe Frauenheilkd 2014;74: 81-88.

55 Bergamaschi G, Di Sabatino A, Albertini R, et al: Prevalence and pathogenesis of anemia in inflammatory bowel disease. Influence of anti-tumor necrosis factor-alpha treatment. Haematologica 2010;95:199-205.

56 Miyazono K, Kamiya Y, Morikawa M: Bone morphogenetic protein receptors and signal transduction. J Biochem 2010;147:35-51.

57 Han X, Zhou DB, Xu CM, et al: Effect of erythropoietin on proinflammatory factors of human monocytes and its mechanisms (in Chinese). Zhongguo Shi Yan Xue Ye Xue Za Zhi 2011;19:738-743.

58 Schwoebel F, van Eijk LT, Zboralski D, et al: The effects of the anti-hepcidin Spiegelmer NOX-H94 on inflammation-induced anemia in cynomolgus monkeys. Blood 2013;121: 2311-2315. 